\title{
Alterations of the Cerebral White Matter in a Middle-Aged Patient with Turner Syndrome: An MRI Study
}

\author{
Haruko Tanji ${ }^{a}$ Katsuo Nakajimab Manabu Wada ${ }^{a}$ \\ Takeo Kato ${ }^{\mathrm{a}}$ \\ a Department of Neurology, Hematology, Metabolism, Endocrinology and \\ Diabetology (DNHMED), Yamagata University School of Medicine, and \\ ${ }^{b}$ Department of Internal Medicine, Tendo City Hospital, Yamagata, Japan
}

\section{Key Words}

Turner syndrome $\cdot$ White matter lesion $\cdot \mathrm{MRI} \cdot \mathrm{KL}-6$

\section{Abstract}

A 52-year-old woman with intellectual disability was admitted to the hospital due to pneumonia. MRI of her brain showed diffuse hyperintensities on $\mathrm{T}_{2}$-weighted and fluid attenuated inversion recovery images in the bilateral cerebral white matter. Laboratory examination revealed sustained high levels of serum KL-6. Karyotyping revealed partial monosomy of the $X$ chromosome. This is the first case showing diffuse white matter lesions in the brain, and sustained high levels of serum KL-6 in Turner syndrome.

\section{Introduction}

Turner syndrome (TS) is caused by partial or complete monosomy X, which is characterized clinically by short stature, estrogen deficiency, increased risk for cardiac abnormalities, and cognitive impairment [1]. Recently, using diffusion tensor imaging and structural imaging methods of MRI, alterations of the cerebral white matter have been demonstrated in young patients with TS [2, 3]. However, on conventional MRI such as $\mathrm{T}_{2}$-weighted (T2W) and fluid attenuated inversion recovery (FLAIR) images, there have been no reports showing extensive white matter changes. One possibility is that white matter changes are too subtle to be detected by conventional MRI. One may speculate that such white matter alterations could be detected by conventional MRI in an advanced stage of the disease. Here, we report a middle-aged woman with TS, whose 
brain MRI showed diffuse hyperintensity on T2W and FLAIR images in the cerebral white matter.

\section{Case Report}

A 52-year-old woman with intellectual disability was admitted to hospital because of cough respiratory distress, and bilateral interstitial shadow on her chest X-ray. Her chest CT revealed micronodular and ground-glass opacity in bilateral lungs. Her blood pressure was 130/75 mm Hg. After the administration of antibiotics, her symptoms and abnormal shadow gradually improved. Bacteriological studies failed to reveal any pathogen.

Intellectual disability had been observed since early infancy; however, no detailed cognitive profiles from the past were available. She was diagnosed at the age of 40 years as having type 2 diabetes mellitus, which has been well controlled without medication. She has been married for approximately 20 years with no children. Neurological examination revealed impairment of skilled movements of the upper extremities and cognitive impairment. She scored 17/30 on the mini-mental state examination. Neuropsychological examination revealed attention deficits, disorientation, impairment of memory and frontal lobe function, and constructional disability. Physical and neurological examinations were otherwise unremarkable.

Her laboratory tests on admission showed a markedly increased KL-6 level of 3,490 U/ml (normal $<500$ ), and hyperglycemia (fasting plasma glucose $150 \mathrm{mg} / \mathrm{dl}$, hemoglobin A1c 5.9\%) without dyslipidemia. Even 9 months after her complete recovery from pneumonia, the level of KL-6 remained high $(1,680 \mathrm{U} / \mathrm{ml})$.

Her neurological abnormalities led us to request MRI of the brain, which showed diffuse hyperintensities on T2W and FLAIR images in the bilateral cerebral white matter (fig. 1a-c). Cerebral MR angiography (MRA) and Doppler ultrasonography revealed no stenotic lesions in the carotid and major cerebral arteries (fig. 1d), and the carotid intima-media thickness was within the normal range $(<1.0 \mathrm{~mm})$. Her ankle brachial index was 1.05 on the right and 0.99 on the left, and funduscopic examination revealed slightly increased light reflex without any hemorrhages or exudates. Chromosomal testing revealed that she had a mosaic karyotype of 45,X (7 cells) and normal 46,XX (23 cells).

\section{Discussion}

We presented a case of a woman with intellectual disability who was diagnosed with mosaic-type TS after her brain MRI showed diffuse hyperintensities on T2W and FLAIR images in the bilateral cerebral white matter. Laboratory tests revealed sustained high KL-6 in serum after her recovery from pneumonia.

To our knowledge, this is the first case of TS showing diffuse hyperintensities in the cerebral white matter on T2W and FLAIR images of MRI. A developmental study in TS has revealed decreased white matter volume in the bilateral occipital lobe [4]. There are several case reports of callosal dysgenesis in patients with TS [5-7]. A recent case report described an intracallosal longitudinal fiber bundle in a woman with TS, which might represent an aberrant cingulum [8]. Holzapfel et al. [2] revealed, using diffusion tensor imaging and structural imaging methods, that 10 young patients (age 7.1-24.4 years; mean $16 \pm 5.12$ ) with TS and 10 age- and gender-matched control subjects exhibited differences in microstructures in white matter. Relative to controls, young females with TS had abnormalities in the deep white matter of the left parietal-occipital region extending anteriorly along the superior longitudinal fasciculus into the deep white matter of the frontal lobe. Moreover, the alterations in white matter morphology 
were correlated with the depressed ability of visuospatial/motor functions observed in TS. These findings suggest that the genes on the $\mathrm{X}$ chromosome might play an important role in morphogenesis of the cerebral white matter. With regard to the present case, one can speculate several possible mechanisms of white matter lesion: (1) the end result of the neurodevelopmental mechanisms which produce subtle white matter abnormalities in younger patients; (2) the lack of X chromosome genes which might result in a TS-specific neurodegenerative process during aging, or (3) the early developmental effects which make women with TS more vulnerable to normal agerelated changes. In any case, it is possible that the white matter changes in TS may develop with aging and may be visualized by conventional MR images, such as T2W and FLAIR images, in later life, as in the present case. Further studies on cerebral white matter lesions in elderly TS patients are needed.

Another possibility is that the white matter hyperintensity on MRI may be ischemic lesions. It has been suggested that TS patients have a metabolic predisposition to ischemia of the brain due to estrogen deficiency $[9,10]$, although there was no information on gonadal steroid levels in our patient. She has had diabetes for years; it is possible that her diabetes may have contributed to the development of diffuse white matter ischemia of the brain. However, this possibility is not supported by the following: (1) the severity of her white matter lesions was classified as grade 3 on the Fazekas rating scale [11], which is too severe for patients without any vascular risk factors except diabetes; (2) her diabetes is mild and well controlled without any medication, and the severity of white matter lesions is not associated with diabetes, as shown in previous studies [12,13]; (3) she had no blood hypertension or dyslipidemia, and (4) she had no atherosclerotic lesions on carotid ultrasonography or on MRA of the brain, and the results of her ankle brachial index and funduscopic examination did not show any signs of apparent peripheral atherosclerotic changes.

This case also showed a sustained high level of KL-6 in serum even 9 months after her recovery from pneumonia. KL-6, a mucin-like high-molecular-weight glycoprotein, has been shown to correlate with activity of interstitial pneumonia [14]. Previous studies showed higher bronchial reactivity and anomalous pulmonary venous return in TS patients $[15,16]$, but there are no reports showing an association between TS and interstitial lung diseases or KL-6. Sun et al. [14] reported that KL-6 was expressed in the lungs of premature neonates and infants with gestational age from 23 to 40 weeks. The sustained high level of KL- 6 in the present case may be related to a possible prematurity of the lungs in TS. Further studies are needed to examine whether serum KL-6 levels are increased in TS.

We reported a case of TS with diffuse hyperintensities on T2W and FLAIR images in the bilateral cerebral white matter, along with sustained elevation of KL-6 in serum after her recovery from pneumonia. Further studies are needed to investigate the relationship between elderly females with TS and white matter lesions. Physicians should carefully investigate patients with metabolic or neuropsychological problems whose brain MRI show diffuse white matter lesions, because patients with mosaic-type TS may remain undiagnosed. 

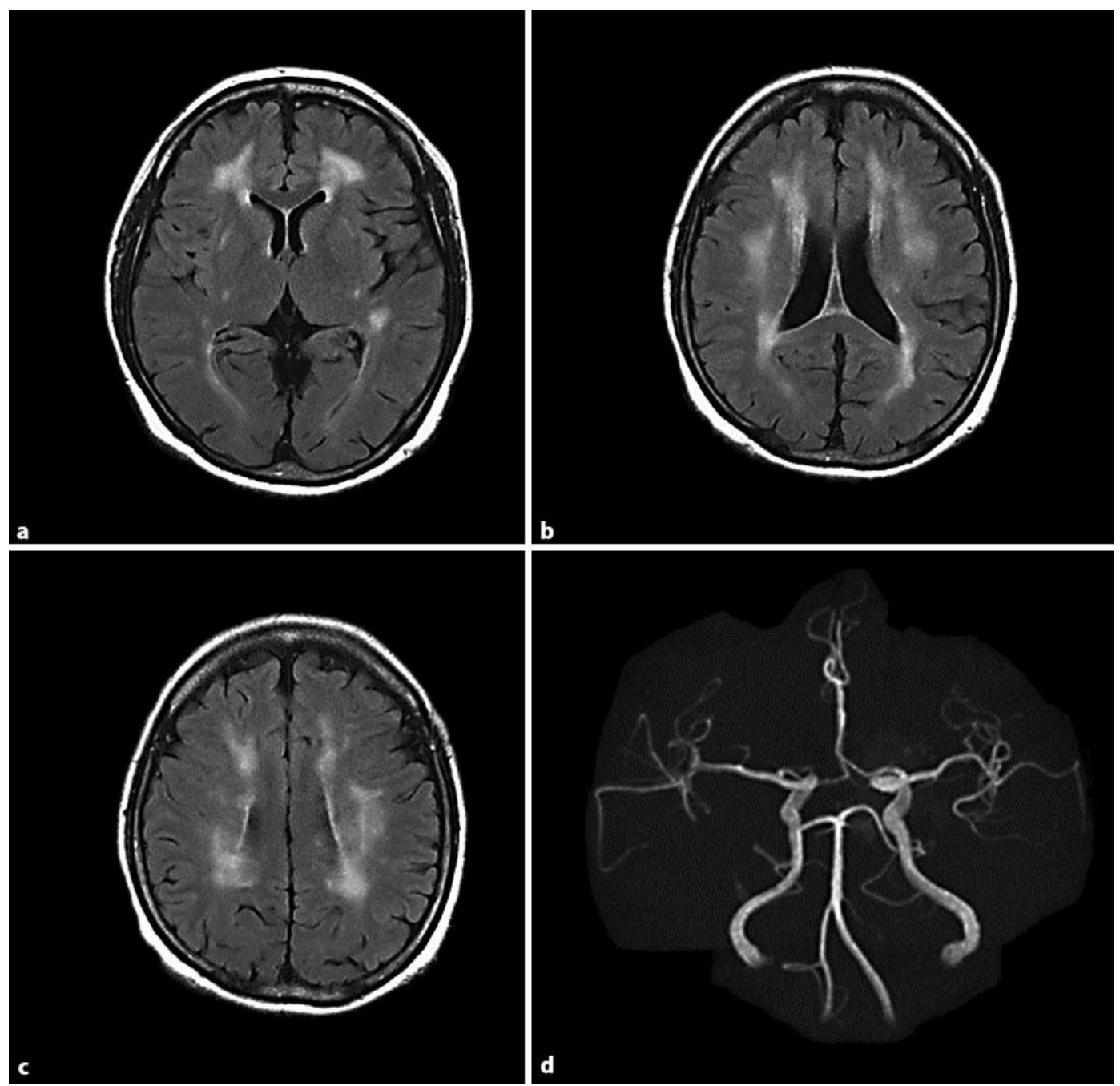

Fig. 1. a-c FLAIR image of the brain shows diffuse hyperintensity in bilateral periventricular and deep cerebral white matter, sparing subcortical white matter. Both anterior and posterior white matters were equally affected and no significant predominance in distribution was noted. $\mathbf{d}$ MRA demonstrates normal intracranial arteries without a finding indicative of atherosclerotic pathology. As an incidental finding, the proximal portion of the anterior cerebral artery is of azygous type.

\section{References}

1 Kesler SR: Turner syndrome. Child Adolesc Psychiatr Clin N Am 2007;16:709-722.

2 Holzapfel M, Barnea-Goraly N, Eckert MA, et al: Selective alterations of white matter associated with visuospatial and sensorimotor dysfunction in Turner syndrome. J Neurosci 2006;26:7007-7013.

3 Yamagata B, Barnea-Goraly N, Marzelli MJ, Park Y, Hong DS, Mimura M, Reiss AL: White matter aberrations in prepubertal estrogen-naive girls with monosomic Turner syndrome. Cereb Cortex 2011, Epub ahead of print.

4 Brown WE, Kesler SR, Eliez S, et al: Brain development in Turner syndrome: a magnetic resonance imaging study. Psychiatry Res 2002;116:187-196.

5 Kimura M, Nakajima M, Yoshino K: Ullrich-Turner syndrome with agenesis of the corpus callosum. Am J Med Genet 1990;37:227-228. 
-6 El Abd S, Patton MA, Turk J, Hoey H, Howlin P: Social, communicational, and behavioral deficits associated with ring X turner syndrome. Am J Med Genet 1999;88:510-516.

7 Hori A: Precocious cerebral development associated with agenesis of the corpus callosum in mid-fetal life: a transient syndrome? Acta Neuropathol 1996;91:120-125.

-8 Sy J, Alturkustani M, Ang LC: Intracallosal longitudinal fiber bundle: an unexpected finding mimicking demyelination in a patient with Turner syndrome. Acta Neuropathol 2010;120:545-547.

9 Irioka T, Mizusawa H: Ischemic stroke in a young patient with Turner syndrome. Neurol Sci 2011;32:317-319.

10 Peper JS, van den Heuvel MP, Mandl RC, Pol HE, van Honk J: Sex steroids and connectivity in the human brain: a review of neuroimaging studies. Psychoneuroendocrinology 2011;36:1101-1113.

11 Fazekas F, Kleinert R, Offenbacher H, Schmidt R, Kleinert G, Payer F, Radner H, Lechner H: Pathologic correlates of incidental MRI white matter signal hyperintensities. Neurology 1993;43:1683-1689.

12 Wada M, Takahashi Y, Iseki C, Kawanami T, Daimon M, Kato T: Plasma fibrinogen, global cognitive function, and cerebral small vessel disease: results of a cross-sectional study in community-dwelling Japanese elderly. Intern Med 2011;50:999-1007.

13 Weinger K, Jacobson AM, Musen G, Lyoo IK, Ryan CM, Jimerson DC, Renshaw PF: The effects of type 1 diabetes on cerebral white matter. Diabetologia 2008;51:417-425.

14 Sun AP, Ohtsuki Y, Fujita J, et al: KL-6, a human MUC1 mucin, is expressed early in premature lung. Respir Med 2003;97:964-969.

15 Villa MP, Bernardi F, Burnaccini M, Tura A, Martelli M, Mazzanti L, Bergamaschi R, Cacciari E: Bronchial reactivity and sex hormone: study in a Turner's po pulation. Pediatr Pulmonol 1990;9:199-205.

16 Counil F, Ichay L, Guillaumont S, Dumas R, Voisin M: Association of severe bronchial disease (bronchial casts, bronchiectasis) and partial abnormal pulmonary venous drainage in 2 children with Turner's syndrome. Arch Pediatr 1999;6:1070-1074. 